\title{
Titrated oxygen requirement and prognostication in idiopathic pulmonary
}

\section{fibrosis}

\author{
J.L. Hook ${ }^{\star, \#}$, S.M. Arcasoy,\#, D. Zemmel ${ }^{\#}$, M.N. Bartels ${ }^{\#, \oplus,}$ \\ S.M. Kawut ${ }^{+, \S, f}$ and D.J. Lederer*,\#,**
}

ABSTRACT: The supplemental oxygen flow rate is a common bedside measure of gas exchange impairment. We aimed to determine whether a titrated oxygen requirement (TOR) predicted mortality in idiopathic pulmonary fibrosis (IPF).

We examined 104 adults with IPF enrolled in a prospective cohort study and a validation cohort of 151 adults with a variety of interstitial lung diseases (ILDs). The TOR was defined as the lowest oxygen flow rate required to maintain an oxyhaemoglobin saturation of $96 \%$ while standing. Cox proportional hazards models and time-dependent receiver operating characteristic curves were used to examine survival time.

A higher TOR was associated with a greater mortality rate independent of forced vital capacity and 6-min walk test results in IPF (adjusted hazard ratio (per $1 \mathrm{~L} \cdot \mathrm{min}^{-1}$ ) 1.16, 95\% $\mathrm{Cl} 1.06-1.27$ ). The TOR was at least as accurate as pulmonary function and 6-min walk testing at predicting 1-yr mortality. Findings were similar in other ILDs.

The TOR is a simple, inexpensive bedside measurement that aids prognostication in IPF.

KEYWORDS: Idiopathic pulmonary fibrosis, interstitial lung diseases, outcome prediction, pulmonary fibrosis, pulmonary gas exchange

$\mathbf{T}$ he interstitial lung diseases (ILDs) are a heterogeneous group of diseases characterised by inflammation and fibrosis of the lung parenchyma [1]. Idiopathic pulmonary fibrosis (IPF), a common ILD that affects older adults, carries a median survival time of $\sim 3$ yrs $[2,3]$. However, a significant fraction of affected individuals survive for $>10$ yrs $[4,5]$. Accurate tools to improve prognostication in IPF would aid clinicians and researchers by informing guidelines for the referral and prioritisation of patients for lung transplantation, facilitating discussions of end-oflife planning and identifying appropriate candidates for experimental therapies.

A number of studies have identified associations between reduced exercise test performance and higher mortality rates in IPF [4-11]. For example, both reduced maximal exercise capacity during cycle ergometry $[5,7,11]$ and a shorter distance walked during hallway walk testing are associated with higher mortality rates [8-10]. Well-designed studies have indicated that exertional desaturation during 6-min walk testing may explain the association between decreased exercise capacity and mortality in IPF $[4,6]$.

Exercise capacity and exertional desaturation, however, are strongly influenced by the fraction of inspired oxygen administered during testing [12, 13]. Current guidelines suggest that 6-min walk testing be performed using the patient's "standard rate" $^{\prime \prime}$ of oxygen flow [14]. Previous studies have excluded those with low resting oxyhaemoglobin saturation (arterial oxygen saturation measured by pulse oximetry $\left(S p, \mathrm{O}_{2}\right)$ ) and have administered supplemental oxygen in a nonstandardised fashion during 6-min walk testing [4, 6, 8-10], limiting the widespread application and interpretation of submaximal exercise test results in this population.

The amount of supplemental oxygen required to maintain $\mathrm{Sp}_{\mathrm{p}} \mathrm{O}_{2}$ at physiological levels is a common bedside measure of disease severity across a wide spectrum of lung diseases, and the degree of gas exchange impairment at rest is linked to higher mortality rates in IPF [4, 5, 15-17]. However, there are no data examining the reliability and predictive validity of a standardised measure of
AFFILIATIONS

Depts of *Medicine, and

"Rehabilitation and Regenerative

Medicine, College of Physicians and

Surgeons,

**Dept of Epidemiology, Mailman

School of Public Health, Columbia

University,

\# New York Presbyterian Lung

Transplant and Interstitial Lung

Disease Programs, New York

Presbyterian Hospital/Columbia, New

York, NY,

+Penn Cardiovascular Institute,

${ }^{\S}$ Dept of Medicine, and

${ }^{f}$ Center for Clinical Epidemiology

and Biostatistics, University of

Pennsylvania School of Medicine,

Philadelphia, PA, USA.

CORRESPONDENCE

D.J. Lederer

Division of Pulmonary, Allergy and Critical Care Medicine

College of Physicians and Surgeons

Columbia University

622 W 168th Street

$\mathrm{PH}-14$

Room 104

New York

NY 10032

USA

E-mail: davidlederer@columbia.edu

Received:

June 232011

Accepted after revision:

Aug 162011

First published online:

Sept 012011 
oxygen requirement in IPF, limiting the ability to examine oxygen requirement in epidemiological studies and clinical trials. At our centre, we implemented an oxygen titration protocol to standardise the use of supplemental oxygen during 6-min walk testing in 2007. In the current study, we hypothesised that the oxygen flow rate required to maintain $\mathrm{Sp}_{2} \mathrm{O}_{2}$ at $\geqslant 96 \%$ would predict short-term outcomes in IPF.

\section{METHODS}

\section{Study subjects and study design}

We examined 104 adults with IPF who enrolled in a prospective cohort study of ILD patients at the New York Presbyterian Lung Transplant and Interstitial Lung Disease Programs (New York Presbyterian Hospital/Columbia, New York, NY, USA) between February 2007 and June 2010. We screened 505 adults seen by a pulmonologist at our centre with suspected or known ILD (fig. 1). Of these, 418 consented and 151 met American Thoracic Society/European Respiratory Society criteria for IPF [18]. We excluded 262 who met criteria for an ILD other than IPF, such as those with evidence of connective tissue disease, a history of occupational or environmental exposures known to cause pneumoconioses and those with suspected drug-induced lung disease. We excluded 47 who did not undergo oxygen titration testing at our centre. The most common reasons for not undergoing testing at our centre were: patient preference $(n=17)$, testing not ordered $(n=12)$ and testing performed according to a clinical trial protocol $(n=7)$. The study cohort consisted of 104 participants with IPF who underwent an oxygen titration study at our centre (fig. 1). The Columbia University Medical Center Institutional Review Board (New York, NY)

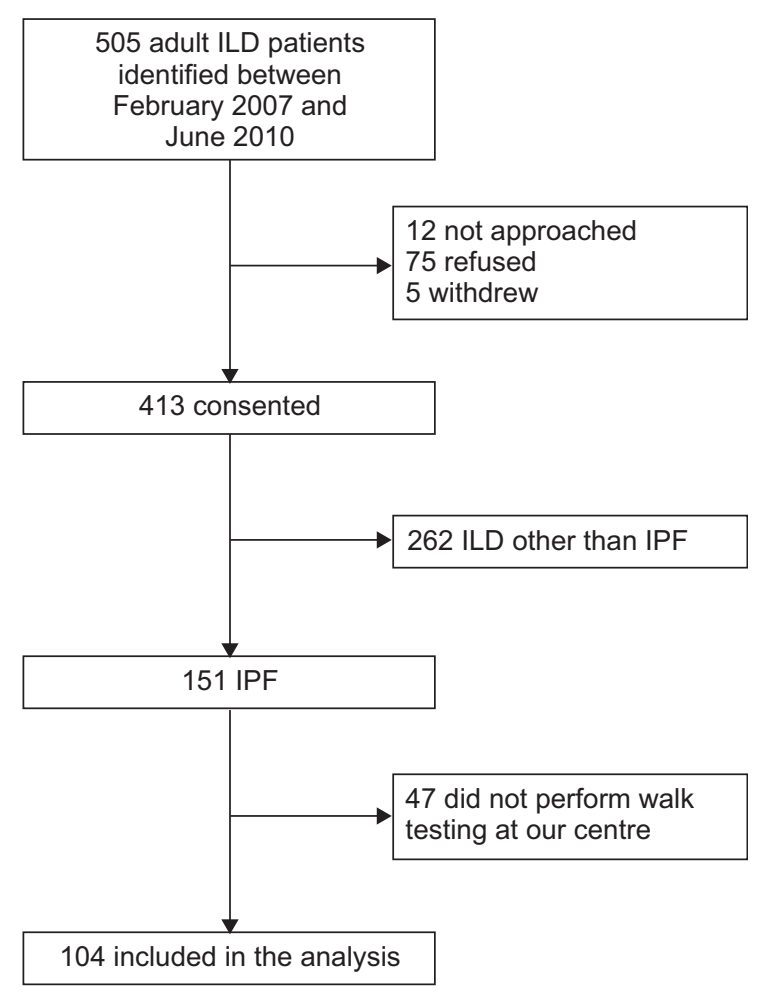

FIGURE 1. Study participant flow. ILD: interstitial lung disease; IPF: idiopathic pulmonary fibrosis. approved the prospective study. All participants gave written informed consent.

To validate our findings, we subsequently assembled a cohort of 152 ILD patients not enrolled in the prospective study by searching the Clinical Data Warehouse at Columbia University (New York, NY) for all 6-min walk tests performed for adults with ILD between February 2007 and April 15, 2010. We excluded one patient who did not have available spirometry data. The Columbia University Medical Center Institutional Review Board approved this retrospective study and waived informed consent.

\section{6-min walk testing and titrated oxygen requirement}

6-min walk testing was performed in a 30-m (100-ft), straight indoor hallway by a single physiotherapist in accordance with American Thoracic Society guidelines [14]. Supplemental oxygen was administered according to the results of the oxygen titration study described below using a commercially available integrated E-cylinder, valve and regulator device (Linde Integrated Valve; Linde North America, Murray Hill, NJ, USA). Pulse oximetry was performed prior to the 6-min walk test and during patientinitiated rests using a single Nellcor OmiMax N-65 pulse oximeter (Covidien-Nellcor, Boulder, CO, USA). A single pulse oximeter was selected in order to minimise measurement variability. Pulse oximetry was not monitored during ambulation. Distance walked during testing (6-min walk distance (6MWD)), oxyhaemoglobin saturation at the end of the test (end-walk $\mathrm{Sp}_{\mathrm{p}} \mathrm{O}_{2}$ ) and modified Borg dyspnoea score were measured after each walk. Testing was not terminated for desaturation $<80 \%$.

Immediately prior to 6-min walk testing, an oxygen titration study was performed to determine the lowest oxygen flow rate required to maintain an $\mathrm{Sp}, \mathrm{O}_{2}$ of $\geqslant 96 \%$ in the standing position (titrated oxygen requirement (TOR)). Titration began with the patient breathing room air. $\mathrm{Sp}, \mathrm{O}_{2}$ was monitored for $1 \mathrm{~min}$. If the $\mathrm{Sp}_{\mathrm{P}} \mathrm{O}_{2}$ was $\geqslant 96 \%$, the study ended. If the $\mathrm{Sp}, \mathrm{O}_{2}$ was $<96 \%$, the oxygen flow rate was increased each minute to achieve a target $\mathrm{Sp}_{\mathrm{O}} \mathrm{O}_{2}$ of $\geqslant 96 \%$ using the following titration steps: $1,2,3,4,5,6,8$, 12 and $15 \mathrm{~L} \cdot \mathrm{min}^{-1}$. A nasal cannula was used to administer 1$6 \mathrm{~L} \cdot \mathrm{min}^{-1}$ and a non-rebreather mask was used for $8-15 \mathrm{~L} \cdot \mathrm{min}^{-1}$. To examine the interobserver reliability (precision) of the TOR, two investigators measured the TOR of 10 study participants with IPF in a blinded fashion $<7$ days apart. The results were identical for nine out of 10 participants and disagreed by only $1 \mathrm{~L} \cdot \mathrm{min}^{-1}$ in the remaining participant. The weighted kappa (a test of agreement between groups for ordinal variables, such as TOR) was 0.90 , suggesting that the TOR is a reliable measure. 6-min walk testing was performed using the TOR; no further changes were made to oxygen flow during 6-min walk testing. We have performed 6-min walk testing using the titrated oxygen flow rate in $>300$ patients with ILD with no adverse events other than dyspnoea.

\section{Analysis}

The cohort was divided into rough quartiles of TOR. We examined associations between TOR and the rate of death using Cox proportional hazards models censored at the time of transplantation. Stratified Cox models with strata for diagnostic category were used in the validation cohort in order to account for variation by diagnosis. We included purposefully selected covariates known to be important prognostic factors in IPF: age, $6 \mathrm{MWD}$, end-walk $\mathrm{Sp}_{\mathrm{p}} \mathrm{O}_{2}$ and forced vital capacity (FVC). We 
examined the ability of TOR and other established prognostic factors to predict the risk of death and transplantation using time-dependent receiver operating characteristic (ROC) curves (survivalROC package in $R$ ) [19]. Statistical analyses were performed using SAS version 9.1 (SAS Institute, Cary, NC, USA), and $R$ version 2.8.1 (R Foundation, Vienna, Austria). p-values $<0.05$ were considered statistically significant.

\section{RESULTS}

\section{IPF participant characteristics}

The mean \pm SD age of the study participants was $62 \pm 7$ yrs and $79 \%$ were male. $45 \%$ had diagnoses confirmed by surgical lung biopsy. FVC was $54 \pm 21 \%$ predicted and diffusing capacity of the lung for carbon monoxide (DL,CO) was $37 \pm 13 \%$ pred. $6 \mathrm{MWD}$ was $394 \pm 133 \mathrm{~m}$ and $56 \%$ had an end-walk $\mathrm{Sp}_{\mathrm{p}, \mathrm{O}_{2}}$ of $\leqslant 88 \%$ (interquartile range (IQR) 82-92\%). Compared with those who performed an oxygen titration study, excluded participants were older (mean age 68 versus 62 yrs; $p<0.001$ ), more frequently female (38 versus $21 \% ; \mathrm{p}=0.03$ ) and had lower FVC (mean 46 versus $54 \%$ pred; $\mathrm{p}=0.046$ ).

The median TOR was $2 \mathrm{~L} \cdot \mathrm{min}^{-1}\left(\mathrm{IQR} \quad 0-4 \mathrm{~L} \cdot \mathrm{min}^{-1}\right.$ ) and the mean \pm SD TOR was $3.3 \pm 4.6 \mathrm{~L} \cdot \mathrm{min}^{-1}$. Other than shortness of breath, no adverse events occurred during 6-min walk testing or oxygen titration. Those with higher TOR values were more likely to be male, and tended to have lower FVC, lower total lung capacity, lower $\mathrm{DL}, \mathrm{CO}$, higher pulmonary artery pressure and lower 6MWD (table 1).

\section{Associations of TOR with survival time in IPF}

During a median follow-up time of 11 months (IQR 5-21 months), 17 study participants died without undergoing lung transplantation and 35 underwent lung transplantation. Figure 2a shows unadjusted survival by TOR group. After adjustment for age, $6 \mathrm{MWD}$, end-walk $S \mathrm{p}, \mathrm{O}_{2}$, and $\mathrm{FVC}$, greater TOR was associated with a higher mortality rate (table 2). For example, after adjustment for potential confounders, a TOR of $\geqslant 8 \mathrm{~L} \cdot \mathrm{min}^{-1}$ was associated with a 6.7 -fold increased mortality rate $(95 \% \mathrm{CI}$ $1.7-25 ; \mathrm{p}=0.005)$ compared with a TOR of $0 \mathrm{~L} \cdot \mathrm{min}^{-1}$. Examination of TOR as a quasicontinuous variable showed a similar finding, with an adjusted hazard ratio (HR) for death of 1.16 (95\% CI 1.061.27; $\mathrm{p}=0.001)$ per $1-\mathrm{L} \cdot \mathrm{min}^{-1}$ increase in TOR. Survival time was shorter for those with a TOR of $\geqslant 1 \mathrm{~L} \cdot \mathrm{min}^{-1}$ compared with those with a TOR of $0 \mathrm{~L} \cdot \mathrm{min}^{-1}$ (multivariable-adjusted HR 3.2, 95\% CI 1.11-9.3; $\mathrm{p}=0.003$ ) (online supplementary fig. E1).

After adjusting for age, TOR and FVC (model 2 in table 2), each $100-\mathrm{m}$ decrement in 6MWD was associated with a two-fold higher mortality rate (HR 2.1, 95\% CI 1.4-3.0; $\mathrm{p}<0.001$ ), but endwalk $\mathrm{Sp}, \mathrm{O}_{2}$ was not associated with the risk of death $(\mathrm{p}=0.32)$. Similarly, an end-walk $\mathrm{Sp}_{\mathrm{p}} \mathrm{O}_{2}<88 \%$ was associated with a nonsignificant $80 \%$ increase in mortality rate (adjusted HR 1.8, 95\% CI 0.6-5.4; $\mathrm{p}=0.31$ ).

\section{Prediction of death at $1 \mathrm{yr}$ in IPF}

Time-dependent ROC curves for the prediction of death at $1 \mathrm{yr}$ using TOR and other established prognostic factors are shown in figure 3a. The areas under the curves (AUCs) for TOR were 0.75 for death; this was similar to or greater than the AUCs for FVC, $D \mathrm{~L}, \mathrm{CO}, 6 \mathrm{MWD}$ and end-walk $\mathrm{Sp}_{\mathrm{p}} \mathrm{O}_{2}$, which ranged from 0.60 to 0.70. A TOR of $\geqslant 1 \mathrm{~L} \cdot \mathrm{min}^{-1}$ was $89 \%$ sensitive and $62 \%$ specific for death within $1 \mathrm{yr}$, yielding positive- and negative-likelihood ratios of 2.34 and 0.18 , respectively (table 3 ). Table 3 shows the positive and negative predictive values for three TOR thresholds calculated using hypothetical low (10\%), intermediate (50\%) and high $(90 \%)$ pre-test event probabilities. A TOR of $0 \mathrm{~L} \cdot \mathrm{min}^{-1}$

\section{TABLE 1 Participant characteristics in the derivation cohort}

\begin{tabular}{|c|c|c|c|c|c|}
\hline & \multirow[t]{2}{*}{ Participants n } & \multicolumn{4}{|c|}{ TOR L· $\min ^{-1}$} \\
\hline & & 0 & $1-2$ & 3-6 & 8-15 \\
\hline Participants n & 104 & 42 & 20 & 28 & 14 \\
\hline Age yrs & 104 & $62 \pm 12$ & $62 \pm 6$ & $62 \pm 7$ & $64 \pm 4$ \\
\hline Males \% & 104 & 79 & 70 & 79 & 93 \\
\hline Body mass index $\mathrm{kg} \cdot \mathrm{m}^{-2}$ & 104 & $28 \pm 5$ & $30 \pm 6$ & $28 \pm 4$ & $30 \pm 5$ \\
\hline Ever-smokers \% & 104 & 71 & 70 & 61 & 64 \\
\hline FVC $\%$ pred & 104 & $65 \pm 21$ & $45 \pm 17$ & $44 \pm 16$ & $50 \pm 16$ \\
\hline Total lung capacity $\%$ pred & 103 & $67 \pm 18$ & $48 \pm 11$ & $49 \pm 9$ & $53 \pm 10$ \\
\hline DL,Co \% pred & 101 & $45 \pm 13$ & $37 \pm 8$ & $33 \pm 11$ & $27 \pm 10$ \\
\hline \multicolumn{6}{|c|}{ Resting supplemental oxygen prescription } \\
\hline$L \cdot \min ^{-1}$ & 104 & $0(0-0)$ & $2(0-2.5)$ & $3(2-4)$ & $3.5(0-4)$ \\
\hline Room air ${ }^{\#}$ & 104 & 79 & 40 & 18 & 43 \\
\hline $\bar{P}_{\mathrm{pa}}{ }^{\top} \mathrm{mmHg}$ & 83 & $19 \pm 4$ & $22 \pm 4$ & $23 \pm 7$ & $27 \pm 11$ \\
\hline PVR Wood units & 76 & $2.3 \pm 0.7$ & $2.5 \pm 0.7$ & $3.2 \pm 1.3$ & $3.0 \pm 1.4$ \\
\hline 6MWD m & 104 & $456 \pm 134$ & $372 \pm 133$ & $335 \pm 101$ & $357 \pm 119$ \\
\hline End-walk $\mathrm{Sp}, \mathrm{O}_{2} \%$ & 104 & $89 \pm 7$ & $84 \pm 7$ & $83 \pm 7$ & $92 \pm 6$ \\
\hline
\end{tabular}

Data are presented as mean \pm SD or median (interquartile range), unless otherwise stated. End-walk arterial oxygen saturation measured by pulse oximetry (Sp, $\mathrm{O}_{2}$ ) represents oxyhaemoglobin saturation at the end of 6 -min walk testing. TOR: titrated oxygen requirement; FVC: forced vital capacity; \% pred: \% predicted; DL,CO: diffusing capacity of the lung for carbon monoxide; $\bar{P}$ pa: mean pulmonary artery pressure; PVR: pulmonary vascular resistance; $6 \mathrm{MWD}$ : 6 -min walk distance. ${ }^{\#}: 0 \mathrm{~L} \cdot$ min $^{-1}$; ? : missing for 14 participants in the $0 \mathrm{~L} \cdot \mathrm{min}^{-1}$ group, three in the $1-2 \mathrm{~L} \cdot \mathrm{min}^{-1}$ group, two in the $3-6 \mathrm{~L} \cdot \mathrm{min}^{-1} \mathrm{group}$ and two in the $8-15 \mathrm{~L} \cdot \mathrm{min}^{-1} \mathrm{group}$. 


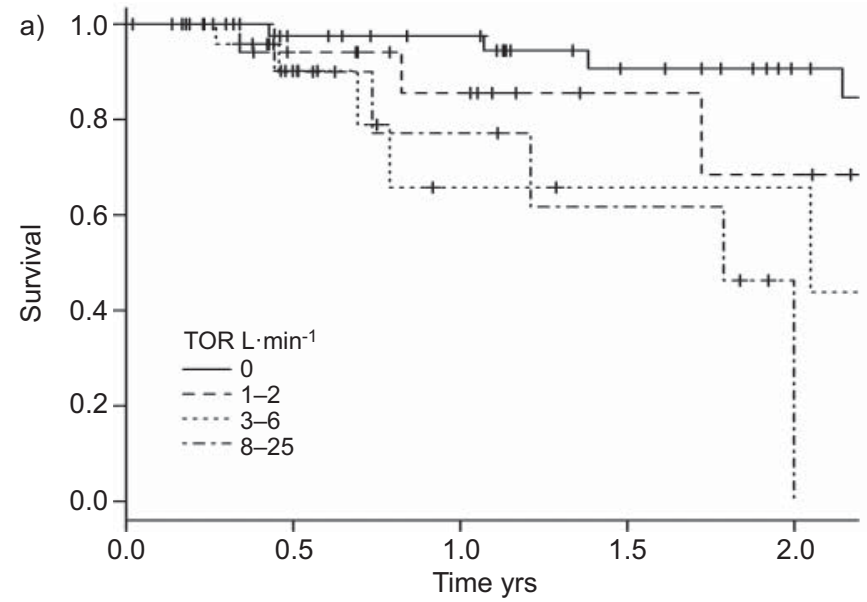

b)

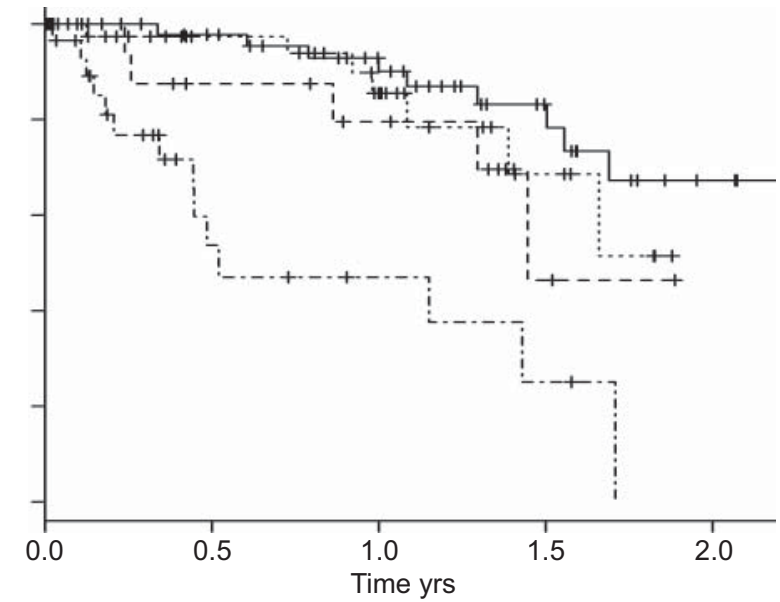

FIGURE 2. Unadjusted survival in the a) derivation ( $p$-value for trend $($ ptrend $)=0.001)$ and b) validation (ptrend<0.001) cohorts. TOR: titrated oxygen requirement.

combined with a low pre-test probability of death had a $98 \%$ negative predictive value at $1 \mathrm{yr}$.

\section{Validation cohort}

The validation cohort consisted of 151 patients: 46 patients with connective tissue disease-associated ILD, 19 with IPF (who were not included in the original cohort), 27 with idiopathic nonspecific interstitial pneumonia and 59 with other forms of ILD (14 with hypersensitivity pneumonitis, 15 with uncharacterised ILD, 11 with pulmonary sarcoidosis, five with lymphangioleiomyomatosis, three with ILD due to prior chemotherapy, three with alveolar proteinosis, two with asbestosis, two with silicosis, and one each with Hermansky-Pudlak syndrome, idiopathic pleuroparenchymal fibroelastosis, pulmonary Langerhans' cell histiocytosis and neurofibromatosis). Associations of TOR with survival time and prediction of survival time in the validation cohort were similar but of smaller magnitude than in the derivation cohort (tables 2 and 3, figs $2 b$ and $3 b$, and online supplementary fig. E2). For example, the multivariable-adjusted
HR for death was 1.10 (95\% CI 1.01-1.18; $\mathrm{p}=0.02)$ in the validation cohort compared with 1.16 in the derivation cohort.

\section{DISCUSSION}

We have shown that the supplemental oxygen flow rate required to maintain an $\mathrm{Sp}_{\mathrm{p}} \mathrm{O}_{2}$ of $\geqslant 96 \%$ at rest predicts 1 -yr outcomes at least as well as established measures of lung mechanics (FVC), gas exchange ( $\mathrm{D} \mathrm{L}, \mathrm{CO}$ and end-walk $\mathrm{Sp}_{\mathrm{p}} \mathrm{O}_{2}$ ) and exercise capacity (6MWD) in patients with IPF and other forms of ILD. Death was uncommon among those with a TOR of $0 \mathrm{~L} \cdot \mathrm{min}^{-1}$ and higher TOR values had greater specificity for the prediction of death. 6-min walk testing using a supplemental oxygen flow rate determined by this novel titration protocol is safe.

The prognostic importance of supplemental oxygen requirements in ILD has previously been examined. EGAN et al. [20] used Organ Procurement and Transplantation Network data to examine the association between the "oxygen requirement at rest" and the risk of dying while on a waiting list for lung transplantation among 608 ILD patients during the development

TABLE 2 Associations of titrated oxygen requirement (TOR) with survival time

\begin{tabular}{|c|c|c|c|c|c|c|c|}
\hline & \multicolumn{5}{|c|}{ TOR L. $\min ^{-1}$} & \multicolumn{2}{|c|}{ HR per $1-L \cdot \min ^{-1}$ increase in TOR } \\
\hline & 0 & $1-2$ & $3-6$ & $8-15^{\#}$ & ptrend & HR $(95 \% \mathrm{Cl})$ & p-value \\
\hline \multicolumn{8}{|l|}{ Derivation cohort $\pi$} \\
\hline Unadjusted HR (95\% Cl) & Ref. & $3.7(1.1-13)$ & $5.3(1.6-18)$ & $7.5(2.1-27)$ & 0.003 & $1.14(1.06-1.24)$ & $<0.001$ \\
\hline \multicolumn{8}{|l|}{ Adjusted HR (95\% Cl) } \\
\hline \multicolumn{8}{|l|}{ Validation cohort ${ }^{+}$} \\
\hline Unadjusted HR (95\% Cl) & Ref. & $2.2(0.7-7.2)$ & $1.2(0.4-3.3)$ & $7.7(2.7-22)$ & $<0.001$ & $1.13(1.06-1.20)$ & $<0.001$ \\
\hline \multicolumn{8}{|l|}{ Adjusted HR $(95 \% \mathrm{Cl})$} \\
\hline Model 1 & Ref. & $1.4(0.4-5.4)$ & $0.7(0.2-2.4)$ & $3.8(1.01-15)$ & 0.01 & $1.10(1.01-1.19)$ & 0.02 \\
\hline Model 2 & Ref. & $1.4(0.4-5.3)$ & $0.7(0.2-2.3)$ & $3.9(1.05-15)$ & 0.01 & $1.10(1.01-1.18)$ & 0.02 \\
\hline
\end{tabular}

Model 1 was adjusted for age, 6-min walk distance and oxygen saturation at the end of the 6-min walk test. Model 2 is model 1 plus adjustment for forced vital capacity \%

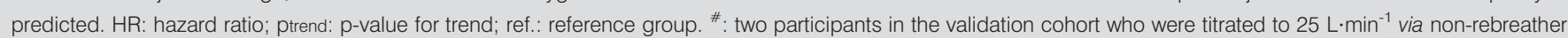
mask were included in this group; $": n=104 ;{ }^{+}: n=151$. 

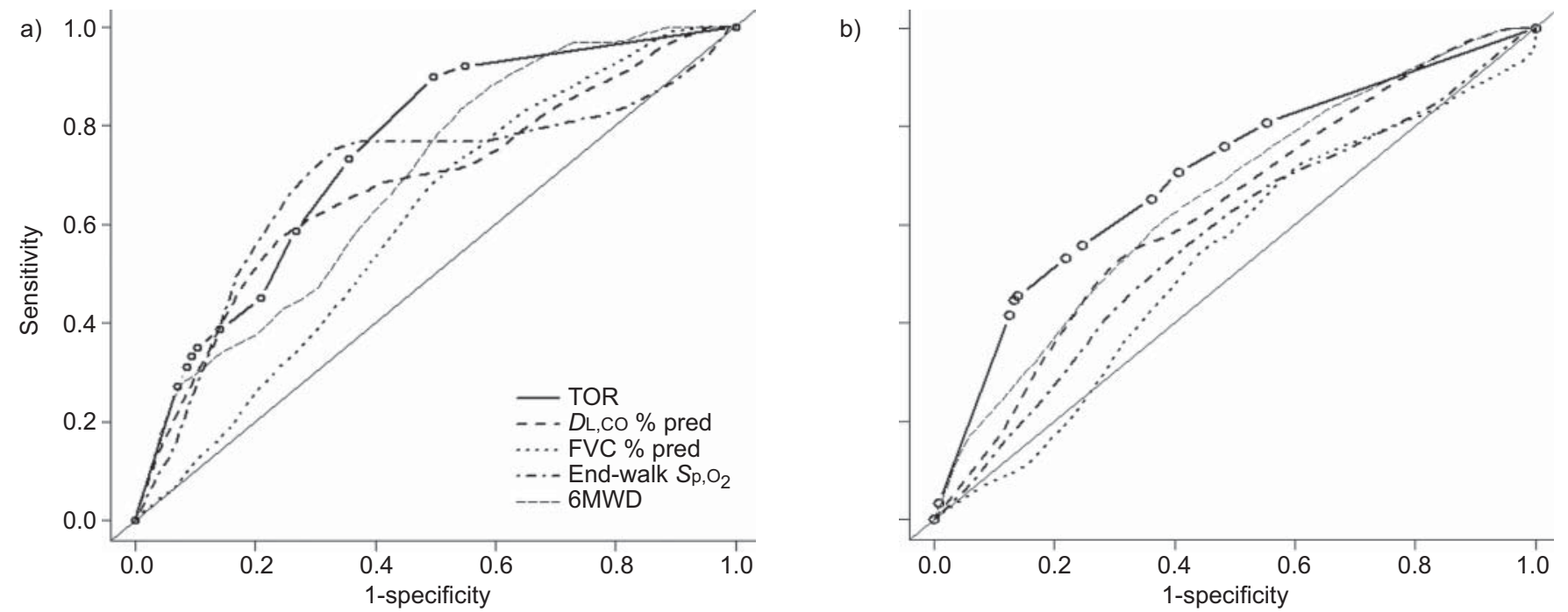

FIGURE 3. Time-dependent receiver operating characteristic curves for the prediction of death within $1 \mathrm{yr}$ in the a) derivation and b) validation cohorts. The area under the curve (AUC) for titrated oxygen requirement (TOR) was 0.75 in the derivation cohort and 0.70 in the validation cohort. The AUC for the other four variables ranged from 0.60 to 0.70 in the derivation cohort and 0.53 to 0.65 in the validation cohort. DL,CO: diffusing capacity of the lung for carbon monoxide; \% pred: \% predicted; FVC: forced vital capacity; $\mathrm{Sp}, \mathrm{O}_{2}$ : arterial oxygen saturation measured by pulse oximetry; 6MWD: 6-min walk distance.

of the lung allocation score (LAS). Although those investigators ultimately included oxygen use in the waiting list urgency score of the lung allocation score, they reported that the supplemental oxygen flow rate was not significantly associated with a higher mortality rate in ILD in a multivariable-adjusted model [20]. The discrepancy between their results and ours may be due to a number of factors. First, we used a standardised assessment of oxygen requirement, rather than relying on flow rates reported by transplant centre personnel, which could have been prone to misclassification bias. Secondly, since we did not restrict our analysis to those placed on the waiting list, our cohort may have been more heterogeneous with regard to disease severity, easing detection of an association with mortality. Thirdly, the multivariable-adjusted model used in the LAS may have included factors that explain the association between oxygen flow rate and mortality, such as pulmonary hypertension [21].
Only $80 \%$ of our study subjects underwent right heart catheterisation, limiting our ability to examine pulmonary hypertension as a confounder. Even if pulmonary hypertension were to explain our findings, TOR would still be a clinically useful test, just as $\mathrm{DL}, \mathrm{CO}[4,16,17,22,23]$, end-walk $\mathrm{Sp}_{\mathrm{O}} \mathrm{O}_{2}[4,6]$ and heart rate recovery [24] remain practical clinical tests that are influenced by pulmonary haemodynamics.

Our results suggest that both TOR and 6MWD are each independently associated a higher risk of death in IPF. In contrast to previous studies $[4,6]$, however, we did not detect an association between end-walk $\mathrm{Sp}, \mathrm{O}_{2}$ and the risk of death, possibly due to the administration of higher concentrations of oxygen during walk testing using our protocol. Our approach was to measure and account for supplemental oxygen use, walk distance and desaturation in the same survival models, permitting an

\begin{tabular}{|c|c|c|c|c|c|c|c|c|c|c|}
\hline & \multirow[t]{2}{*}{ Sensitivity \% } & \multirow[t]{2}{*}{ Specificity \% } & \multirow[t]{2}{*}{ LR+ } & \multirow[t]{2}{*}{ LR- } & \multicolumn{2}{|c|}{$\begin{array}{l}\text { Pre-test probability } \\
\qquad 10 \%\end{array}$} & \multicolumn{2}{|c|}{$\begin{array}{l}\text { Pre-test probability } \\
\qquad 50 \%\end{array}$} & \multicolumn{2}{|c|}{$\begin{array}{c}\text { Pre-test probability } \\
90 \%\end{array}$} \\
\hline & & & & & PPV \% & NPV \% & PPV \% & NPV \% & PPV \% & NPV \% \\
\hline \multicolumn{11}{|c|}{ Derivation cohort } \\
\hline$\geqslant 6 \mathrm{~L} \cdot \mathrm{min}^{-1}$ & 44 & 89 & 4.00 & 0.63 & 31 & 93 & 80 & 61 & 97 & 15 \\
\hline $15 \mathrm{~L} \cdot \mathrm{min}^{-1}$ & 22 & 94 & 3.67 & 0.83 & 29 & 92 & 79 & 55 & 97 & 12 \\
\hline \multicolumn{11}{|c|}{ Validation cohort } \\
\hline$\geqslant 1 \mathrm{~L} \cdot \mathrm{min}^{-1}$ & 81 & 45 & 1.46 & 0.43 & 14 & 95 & 59 & 70 & 93 & 20 \\
\hline$\geqslant 6 \mathrm{~L} \cdot \mathrm{min}^{-1}$ & 55 & 86 & 3.96 & 0.52 & 31 & 95 & 80 & 66 & 97 & 18 \\
\hline
\end{tabular}

LR+: positive-likelihood ratio; LR-: negative-likelihood ratio; PPV: positive predictive value; NPV: negative predictive value. 
independent examination of the impact of each factor. Future studies may help elucidate ideal walk testing practices in order to maximise our ability to predict future events.

Practices of referral, listing and prioritisation for lung transplantation might be improved by standardising the measurement and reporting of supplemental oxygen use. Current guidelines recommend that all patients with IPF be referred for evaluation for lung transplantation at the time of diagnosis. If our findings are validated in future studies, it may be reasonable to incorporate a TOR threshold in referral guidelines. It may also be appropriate to implement our titration protocol at lung transplant centres to standardise reporting of supplemental oxygen use.

It may be that measures of gas exchange (e.g. TOR, end-walk $S \mathrm{p}, \mathrm{O}_{2}$ and $D \mathrm{~L}, \mathrm{CO})$ are better predictors of outcome than measures of lung mechanics (e.g. reduced FVC) [4, 6, 10, 25]. TOR, in particular, may have certain strengths that merit its continued investigation and, perhaps, clinical use. For example, in contrast to pulmonary function testing and 6-min walk testing, TOR is inexpensive (and in many settings cost free) and quick (often $<3 \mathrm{~min}$ ). TOR also standardises a widespread clinical practice of gauging disease severity by oxygen requirement. Clinical trialists should consider including TOR as a potential surrogate end-point in studies of novel therapies.

Our findings were somewhat weaker in the validation cohort compared with the derivation cohort. This may be a result of the inclusion of participants with a variety of different ILDs in the validation cohort despite our attempt to control for diagnosis using statistical methods. In addition, the non-IPF diagnoses comprising the validation cohort typically have lower mortality rates than IPF, possibly limiting our ability to detect small differences in mortality rates. Finally, the IPF cohort was prospectively enrolled, while the validation cohort was assembled retrospectively, which may have introduced selection and information bias.

Our study has several limitations. First, we limited our sampling frame to a single tertiary care referral centre with a lung transplant programme, possibly selecting for those with more severe disease. Nevertheless, we were able to identify a subset with low rates of death and lung transplantation. We also excluded those who did not complete an oxygen titration study at our centre, a group characterised by older age and lower lung function. Application of our findings to those with advanced disease should therefore be done cautiously and should be integrated with clinical judgment. Secondly, since priority for lung transplantation in the USA is, in part, based on oxygen requirements, we were careful to avoid treating transplantation as an event of interest, and instead chose to censor upon lung transplantation. However, informative censoring may have limited our ability to examine death as an isolated event. Thirdly, a number of the factors that influence the oxyhaemoglobin desaturation curve, such as arterial $\mathrm{pH}, 2,3$-diphosphoglycerate and body temperature, could have introduced "noise" into our study. Since this measurement error is unlikely to be differential to the risk of death, such error would tend to weaken the results. Therefore, the actual relationship may be even stronger than that shown. Lastly, our 6-min walk protocol varies from that suggested by the American Thoracic Society guidelines and from other centres' published experience [4, 6, 10]. Differences in exercise test results between our study and others should, therefore, be interpreted with care. Our oxygen titration protocol may provide a useful means to standardise 6-min walk testing across centres.

In summary, we developed a simple, safe, inexpensive oxygen titration study that standardises the administration of supplemental oxygen during 6-min walk testing. The oxygen flow rate required to reach a resting $\mathrm{Sp}_{\mathrm{p}} \mathrm{O}_{2}$ of $\geqslant 96 \%$ may aid in identifying those at high and low risk of death. Our findings support the hypothesis that impaired gas exchange is a clinically useful measure of disease severity in IPF.

\section{SUPPORT STATEMENT}

This work was supported by National Institutes of Health (NIH) grants HL086714 and RR024156, the Robert Wood Johnson Physician Faculty Scholars Program, and the Herbert and Florence Irving Scholar Award. This publication was made possible by grant number KL2 RR024156 from the National Center for Research Resources (NCRR), a component of the NIH, and the NIH Roadmap for Medical Research. Its contents are solely the responsibility of the authors and do not necessarily represent the official view of NCRR or NIH. Information on NCRR is available at the NCRR website. Information on ReEngineering the Clinical Research Enterprise can be obtained from the NIH Roadmap website.

\section{STATEMENT OF INTEREST}

Statements of interest for S.M. Kawut and D.J. Lederer can be found at www.erj.ersjournals.com/site/misc/statements.xhtml

\section{REFERENCES}

1 Gross TJ, Hunninghake GW. Idiopathic pulmonary fibrosis. N Engl J Med 2001; 345: 517-525.

2 Selman M, King TE, Pardo A. Idiopathic pulmonary fibrosis: prevailing and evolving hypotheses about its pathogenesis and implications for therapy. Ann Intern Med 2001; 134: 136-151.

3 King TE, Schwarz MI, Brown K, et al. Idiopathic pulmonary fibrosis: relationship between histopathologic features and mortality. Am J Respir Crit Care Med 2001; 164: 1025-1032.

4 Flaherty KR, Andrei AC, Murray S, et al. Idiopathic pulmonary fibrosis: prognostic value of changes in physiology and sixminute-walk test. Am J Respir Crit Care Med 2006; 174: 803-809.

5 King TE Jr, Tooze JA, Schwarz MI, et al. Predicting survival in idiopathic pulmonary fibrosis: scoring system and survival model. Am J Respir Crit Care Med 2001; 164: 1171-1181.

6 Lama VN, Flaherty KR, Toews GB, et al. Prognostic value of desaturation during a 6-minute walk test in idiopathic interstitial pneumonia. Am J Respir Crit Care Med 2003; 168: 1084-1090.

7 Kawut SM, O'Shea MK, Bartels MN, et al. Exercise testing determines survival in patients with diffuse parenchymal lung disease evaluated for lung transplantation. Respir Med 2005; 99: 1431-1439.

8 Hallstrand TS, Boitano LJ, Johnson WC, et al. The timed walk test as a measure of severity and survival in idiopathic pulmonary fibrosis. Eur Respir J 2005; 25: 96-103.

9 Lederer DJ, Arcasoy SM, Wilt JS, et al. Six-minute-walk distance predicts waiting list survival in idiopathic pulmonary fibrosis. Am J Respir Crit Care Med 2006; 174: 659-664.

10 Lettieri CJ, Nathan SD, Browning RF, et al. The distance-saturation product predicts mortality in idiopathic pulmonary fibrosis. Respir Med 2006; 100: 1734-1741.

11 Fell CD, Liu LX, Motika C, et al. The prognostic value of cardiopulmonary exercise testing in idiopathic pulmonary fibrosis. Am J Respir Crit Care Med 2009; 179: 402-407. 
12 Bye PT, Anderson SD, Woolcock AJ, et al. Bicycle endurance performance of patients with interstitial lung disease breathing air and oxygen. Am Rev Respir Dis 1982; 126: 1005-1012.

13 Harris-Eze AO, Sridhar G, Clemens RE, et al. Oxygen improves maximal exercise performance in interstitial lung disease. Am J Respir Crit Care Med 1994; 150: 1616-1622.

14 American Thoracic Society. ATS Statement: guidelines for the sixminute walk test. Am J Respir Crit Care Med 2002; 166: 111-117.

15 Schwartz DA, Helmers RA, Galvin JR, et al. Determinants of survival in idiopathic pulmonary fibrosis. Am J Respir Crit Care Med 1994; 149: 450-454.

16 Collard HR, King TE, Bartelson BB, et al. Changes in clinical and physiologic variables predict survival in idiopathic pulmonary fibrosis. Am J Respir Crit Care Med 2003; 168: 538-542.

17 Latsi PI, du Bois RM, Nicholson AG, et al. Fibrotic idiopathic interstitial pneumonia: the prognostic value of longitudinal functional trends. Am J Respir Crit Care Med 2003; 168: 531-537.

18 Idiopathic pulmonary fibrosis: diagnosis and treatment. International consensus statement. American Thoracic Society (ATS), and the European Respiratory Society (ERS). Am J Respir Crit Care Med 2000; 161: 646-664.
19 Heagerty PJ, Lumley T, Pepe MS. Time dependent ROC curves for censored survival data and a diagnostic marker. Biometrics 2000; 56: 337-344.

20 Egan TM, Murray S, Bustami RT, et al. Development of the new lung allocation system in the United States. Am J Transplant 2006; 6: 1212-1227.

21 Lettieri CJ, Nathan SD, Barnett SD, et al. Prevalence and outcomes of pulmonary arterial hypertension in advanced idiopathic pulmonary fibrosis. Chest 2006; 129: 746-752.

22 Flaherty KR, Mumford JA, Murray S, et al. Prognostic implications of physiologic and radiographic changes in idiopathic interstitial pneumonia. Am J Respir Crit Care Med 2003; 168: 543-548.

23 Nadrous HF, Pellikka PA, Krowka MJ, et al. Pulmonary hypertension in patients with idiopathic pulmonary fibrosis. Chest 2005; 128: 2393-2399.

24 Swigris JJ, Swick J, Wamboldt FS, et al. Heart rate recovery after 6min walk test predicts survival in patients with idiopathic pulmonary fibrosis. Chest 2009; 136: 841-848.

25 Mogulkoc N, Brutsche MH, Bishop PW, et al. Pulmonary function in idiopathic pulmonary fibrosis and referral for lung transplantation. Am J Respir Crit Care Med 2001; 164: 103-108. 